\title{
Vulnerability Assessment and Spatiotemporal Differentiation of Provinces Tourism Economic System Based on the Projection Pursuit Clustering Model
}

\author{
Xiaohong Yu $\mathbb{D},{ }^{1,2}$ Haiyan $X u \mathbb{D}^{1},{ }^{1}$ and Shengqiao Wang $\mathbb{D}^{3}$ \\ ${ }^{1}$ College of Economics and Management, Nanjing University of Aeronautics and Astronautics, Nanjing 210016, China \\ ${ }^{2}$ College of Humanities and Law, Shanghai Business School, Shanghai 200235, China \\ ${ }^{3}$ College of Business Administration, Shanghai Business School, Shanghai 200235, China
}

Correspondence should be addressed to Shengqiao Wang; shengqiaowang@163.com

Received 4 October 2021; Accepted 1 November 2021; Published 16 November 2021

Academic Editor: Lei Xie

Copyright ( 2021 Xiaohong Yu et al. This is an open access article distributed under the Creative Commons Attribution License, which permits unrestricted use, distribution, and reproduction in any medium, provided the original work is properly cited.

The vulnerability assessment indicator system (VAIS), including the tourism economic sensitivity and respondence, is modified and established in this paper. According to the collected data, during 2014-2018 of the 31 provinces of China, of the tourism economy sensitivity and respondence, the improved comprehensive evaluation projection pursuit clustering (PPC) model is established, and the vulnerability indexes of the 31 provinces are calculated, thus expanding the tourism economic vulnerability assessment methods. Our empirical results show that, during the period of 2014 to 2018, the sensitivity, the respondence, and the vulnerability indexes are unbalanced overall. The tourism economy sensitivity and the respondence show that the spatiotemporal distribution characteristics are high in the east and low in the west. On the contrary, as for the vulnerability, the spatiotemporal distribution characteristics are low in the east and high in the west. Among the 40 indicators, the ratio of industrial solid waste utilized (\%), urbanization rate, and the density of grade highway and railway network $\left(\mathrm{km} / \mathrm{km}^{2}\right)$ have the greatest impact on the respondence, while the proportion of the population affected by natural disasters, the diversification index of industrial structure, and the number of traffic accident casualties have the most significant impact on the sensitivity, which are the indicators that have the greatest impact on vulnerability. Therefore, in order to effectively reduce sensitivity, improve respondence, and thus reduce the vulnerability index of the tourism economy, the provinces should first improve the above-mentioned evaluation indicators with the largest weights. Our research results in this paper enrich the theory of sustainable development of the tourism industry and derive managerial and policy insights for further achieving the high-quality development of the tourism economy.

\section{Introduction}

Vulnerability is an important issue in sustainable and highquality development. It has expanded from geosciences and natural disasters to ecology, economics, management, and other fields [1-6]. Vulnerability mainly involves the concepts of "sensitivity," "adaptability," "respondence," and so on [2, 7-11]. As China has promoted high-quality economic development, tourism has become a "fashion" and daily demand for people's pursuit of a better life. The tourism economy has also become an important part of the highquality development of the regional economy. In 2018, China's domestic tourism revenue exceeded five trillion yuan, with 79.91 million direct and indirect employments, accounting for $10.3 \%$ of the total employment. The added value of related industries has reached 4.15 trillion yuan, accounting for $4.5 \%$ of GDP. Provinces and cities such as Shanghai have formulated relevant policies to further develop the tourism industry and even listed it as a strategic pillar industry. The tourism economy system is highly complex. It requires the comprehensive development and utilization of tourism resources, tourism ecosystem, and tourism-related industries such as accommodation, catering, and sports. Its development is related to regional economic development, social service system, natural resources, and ecological environment. The vulnerability of the tourism 
economic system refers to the fact that the tourism economy is constrained by regional natural resource endowment and the basis of social and economic development, which mainly involves the following two issues. First, the tourism industry is apt to be affected by factors such as ecological resources, economy, society, and medical security. The more developed the tourism industry is, the more sensitive it is. Second, the vulnerability of the tourism economy directly affects the healthy development of tourism in a region and also depends on the ability to deal with the changes of external factors. Therefore, the study of tourism economy vulnerability and its influencing factors is of theoretical and practical significance for promoting the high-quality development of the regional tourism economy.

The research on tourism economy vulnerability started in the 1970s and has become an active research topic in the tourism economy [2,6-22]. The research on the evaluation of tourism economy vulnerability mainly involves two research streams. The first one is to establish a systematic and reasonable evaluation index system. However, the index systems established by different scholars can be quite different. For example, the index systems are composed of only 14 indicators [10, 12, 14] and 24 and 34 indicators [18, 19], respectively. The second research stream aims to determine the indicator weights first or directly applies an appropriate model to comprehensive evaluation. The main methods in the second stream include weighted comprehensive evaluation (WCE) $[12,13,15,17,19]$, set pair analysis (SPA) $[9,10,14,16,18,21,22]$, BPNN model [19], factor analysis (FA) and principal component analysis (PCA) [11], projection pursuit (PPC [23, 24] and PPR [25]) model, and TOPSIS [20]. When such methods as SPA, WCE, TOPSIS, and linear weighted index method are applied, the weights of the evaluation indices must be obtained in advance; otherwise, those weights are assumed to be equal. In theory, when FA and PCA modeling are applied, requirements must be met that the number of samples is 3-5 times greater than the number of indicators and that the sample data (approximately) follow normal distribution so that stable and reliable results can be obtained. When BPNN and PPR models are used, some other methods must be used in advance to obtain reasonably expected results, which is a problem to be solved by the comprehensive evaluation method. Moreover, BPNN and PPR also have the characteristics of "garbage in and garbage out." That is, the reliability and rationality of the modeling results of BPNN [19] and PPR [25] depend on the expected value. In addition, when modeling BPNN, verification samples must be used to monitor the training process in the training process to avoid overtraining; otherwise, the model might have no generalization ability or practical value. Therefore, BPNN modeling can be very difficult and complicated to determine reasonable parameters such as the number of neurons on the hidden layer as well as to judge whether overtraining occurs or not. Consequently, the results vary from person to person and are not unique $[26,27]$.

Therefore, this paper aims to obtain the global optimization of the PPC model based on the judgment criterion of whether the optimization process obtains the real global optimal solution, manages to correctly and reliably apply PPC technology to the comprehensive evaluation of tourism economic vulnerability, obtains the weight of each evaluation indicator, constructs the tourism economy vulnerability evaluation function, and avoids the subjectivity and fuzziness of determining the weights. Thus, the subsequent applications of the PPC model are convenient, and shortcomings of the IEW-SPA method and the BPNN model can be overcome. In addition, it can clearly reveal the relationship between the tourism economy vulnerability and each evaluation indicator. Based on the obtained objective weights, the impact degree and importance ranking of each evaluation indicator on the tourism economy vulnerability can be directly determined, and the obstacle degree can be analyzed. This paper makes a comprehensive evaluation and research on the vulnerability of the tourism economy system in 31 provinces, municipalities, and autonomous regions (hereinafter referred to as provinces) from 2014 to 2018 and clarifies the overall situation, time span, provincial spatial differences, and the main impacting (as well as obstacles) factors related to the vulnerability of China's tourism economy system, so as to provide decision-making and planning guidance for the prevention and control of tourism crisis in China and provinces, as well as the reduction of the vulnerability of the tourism economy system.

The remainder of this paper is organized as follows. In Section 2, literature reviews on tourism economy vulnerability and projection pursuit clustering (PPC) model are described. In Section 3, the comprehensive evaluation system for tourism economy vulnerability is put forward and modified. In Section 4, the samples data are collected, and the comprehensive evaluation PPC models for sensitivity index and respondence index of tourism economy are established and thus the vulnerability index. In Section 5, the results and analysis of the spatiotemporal difference of the sensitivity, respondence, and vulnerability of the tourism economy among provinces are presented. The conclusions and discussions are finally drawn in Section 6.

\section{Literature Review}

This section mainly reviews the literature related to tourism economy vulnerability and the application of the PPC model. The first paper [28] on tourism economy vulnerability in developed and developing countries was published in China in 1989 and without empirical research. In 2013, Li [9] applied the set pair analysis method based on information entropy weight (IEW-SPA) to a comprehensive evaluation of the changes and rules of China's tourism economy vulnerability from 1983 to 2011, defined the concept of vulnerability as sensitivity (including 16 evaluation indicators) and respondence (including 15 evaluation indicators), obtained the indicators' weights by information entropy method, got the evaluation values of sensitivity, respondence, and vulnerability respectively, and then constructed the linear relationship among vulnerability, sensitivity, and respondence. Since then, the IEW-SPA method has been widely used in tourism economy vulnerability assessment $[10,14-16,21,22]$, but there is a nonlinear 
relationship among the vulnerability, sensitivity, and respondence $[12,17,19]$. Although both the linear and nonlinear relationships have shown that vulnerability is positively correlated with sensitivity and negatively correlated with respondence, the differences are obvious. In the nonlinear relationship, the vulnerability index increases significantly with the decrease of respondence, and in the linear relationship, the sensitivity decreases and respondence increases in the same order (but the coefficients are different), while in the nonlinear relationship, the changes are not in the same order. Ma et al. [19] constructed the sensitivity and respondence evaluation index system composed of 17 indicators, respectively. Based on the data of 30 provinces in 2017, through expert research, each evaluation index value is put into one of the three equally spaced intervals (referred to as single index evaluation standard interval), and within each interval, 300 samples are generated through equidistant interpolation, from which 50 samples are randomly selected as test samples, and then the sensitivity evaluation model and the respondence evaluation model with network structure of 16-17-1 are established by using BPNN technology. They used the above-mentioned nonlinear relationship to calculate vulnerability, and the information entropy weight, subjective weight, and combination weight are also obtained, but it is not clear how they set the expected output value of the BPNN model, what is the purpose of obtaining combination weight, or how to normalize the index data. The article did not explain how to prevent overtraining when the BPNN model is constructed. The obtained evaluation values of sensitivity and respondence are very small (less than 0.3 ). In the process of modeling, sensitivity, respondence, and vulnerability are divided into three levels, while in the results and analysis part, sensitivity is divided into four levels, which is obviously inconsistent.

It can be seen from the above analyses that although many exploratory studies have been carried out and some results have been achieved in the comprehensive evaluation of tourism economy vulnerability, there are still many problems to be further addressed.

On the other hand, projection pursuit clustering (PPC) technology can be applied to the modeling of high-dimensional nonlinear and abnormal distribution data [29-35]. The basic concept of PPC modeling is completely consistent with the human thinking mode of comprehensive evaluation, ranking, and classification and has been widely used in the fields of management science, social science, natural science, and so on. PPC model has been applied to vulnerability research in other fields [23-25], and 23 papers in Chinese and English have been published, but some problems still arise in the practical applications described in these papers. The main problems include the following: First, when constructing the PPC model, the adopted radius of density window $R=0.1 S_{z}$ is unreasonable, and a real-coded accelerated genetic algorithm (RAGA) is used for optimization, but the global optimal solution was not obtained. Second, it is to establish a PPR model with too few samples, which is less than the number of evaluation indicators, so it is impossible to establish a stable and reliable PPR model.
Third, Zhang and Huang [33] adopted a dynamic clustering PPC model (namely, DCPP) and used RAGA for optimization, and the objective function used is wrong. The correct objective function is put forward [34]. Fourth, there are 3 papers using the Data Process System (DPS) [36] software, and it is impossible to take the density window radius to be $R=m$, because $R$ of the DPS can only be the times of $S_{z}$, such as $0.20 S_{z}, 0.1 S_{z}$, and $0.05 S_{z}$.

\section{Constructing a Comprehensive Evaluation Index System of Tourism Economy Vulnerability}

Based on the evaluation index system of Ma et al. [19], a vulnerability evaluation index system including tourism economy sensitivity and respondence is constructed by adding six indicators and modifying one indicator as follows:

(1) A total of 20 sensitivity evaluation indicators (namely, S1-S20) are selected: the proportion of tourism earnings to GDP (\%), proportion of tourism foreign exchange earnings to total foreign trade export (\%), elasticity coefficient of tourism growth, proportion of tourism foreign exchange earnings to total tourism earnings (\%), diversification index of industrial structure, proportion of foreign visitors arrivals to total tourists (\%), ratio of dependence on foreign trade (\%), proportion of tourism employees to total employees (\%), registered unemployment rate in urban area (\%), number of traffic accidents (cases), number of traffic accident casualties (persons), traffic accident property loss (10,000 yuan), illiteracy rate (\%), social security and stability (\%), proportion of economic losses from natural disasters (\%), proportion of population affected by natural disasters (\%), forest coverage (\%), percentage of employment in tertiary industry (\%), tourist resident ratio (\%), and proportion of output value of tertiary industry to GDP (\%). Since the "crime rate" data cannot be obtained from the China Law Yearbook and judicial reports, it is expressed by "the number of approved (transferred) arrests (criminal) crimes (suspects)/the number of residents (10000)" obtained from the work reports of the people's procuratorate of the provinces. The industrial diversity index is $S 5=-\sum I_{i} \ln \left(I_{i}\right)$, in which $I_{i}$ is the proportion of the added value of the primary, secondary, and tertiary industries to GDP, thus becoming a positive index.

(2) There are 20 respondence indicators (namely, R1-R20), which are the per capita tourism GDP (10000 yuan), tourism earnings growth rate (\%), tourism labor productivity (\%), tourism resource richness, proportion of hotels and catering service earnings to the tourism earnings (\%), total retail sales of social consumer goods (100 million yuan), proportion of public service expenditure to fiscal expenditure (\%), proportion of education 
expenditure to fiscal expenditure (\%), density of grade highway and railway network $\left(\mathrm{km} / \mathrm{km}^{2}\right)$, popularization rate of mobile phone (set/100 persons), business volume of postal and telecommunication services (100 million yuan), proportion of culture and sports investment (\%), urbanization rate (\%), number of healthcare institutions (unit), harmless disposal rate of municipal waste (\%), ratio of industrial solid waste utilized (\%), growth rate of environmental protection investment (\%), per capita disposable income of urban households (10000 yuan), total amount of foreign investment actually utilized as percentage of GDP (\%), and number of personnel of environmental protection institutions (person).

The 40 indicators listed above constitute the vulnerability evaluation index system, in which the forest coverage rate (S17) is a negative indicator. For vulnerability, all respondence indicators are negative indicators. However, because the respondence is the "denominator" of the vulnerability, these respondence indicators can thus be normalized as positive indicators.

\section{Establishing a Comprehensive Evaluation PPC Model of the Tourism Economy Vulnerability}

4.1. Appropriate Preprocessing of the Indicators' Data. According to the evaluation index system shown in Table 1, a total of 155 observations from 2014 to 2018 are collected (due to the large amount of data, the data is omitted from this paper, but it is available upon request). There are both dynamic indicators such as the tourism earnings growth rate and static indicators such as business volume of postal and telecommunication services. The values of different indicators vary greatly. Therefore, the indicators' original data should be standardized to eliminate the adverse impact on the modeling results caused by different dimensions.

Considering that the values of these indicators may exceed the range of modeling sample data in practical application, the normalization (normalization) preprocessing method with the mean being zero and the variation being 1 is adopted. After the preprocessing described above, only half of the indicators' values are distributed in the range of $[-3,3]$ and follow the normal distribution. For example, as shown in Figure 1(a), which is the histogram of S5, the distribution is obviously skewed.

Since the maximum value of the indicator is greater than 3 (or the minimum value is less than -3 ), it is very likely that the modeling results would be distorted. Therefore, the sigmoid function is used for nonlinear normalization for the above-normalized value. Consequently, the distribution characteristics of most indicators are thus improved, with some indicators' value transformed to nearly follow the normal distribution. For example, Figure 1(b) shows the improved distribution histogram of S5. So far, all indicators' values are transformed to the range of $[0,1]$.
4.2. Principle of Constructing PPC Model. Friedman and Tukey [35] put forward the PPC model. The high-dimensional data are projected to low-dimensional subspaces such as one-dimensional or two-dimensional in the "direction of interest." The optimization results are the projection points (values) of the sample data in low-dimension are forming several classes (clusters) as separate as possible and as close as possible in one class. In this paper, an improved projection pursuit index function based on a onedimensional PPC model is constructed as follows:

$$
\begin{array}{r}
Q(a)=\max \left\{\frac{S(a) \cdot D(a)}{k_{4}}\right\}, \\
\text { s.t. } \quad \sum_{j=1}^{m} a^{2}(j)=1, \quad a(j) \in[-1,1] .
\end{array}
$$

In (1), the sample projection values $z(i)=\sum_{j=1}^{m} a(j) x(i, j), a(j)$ is the optimal weight of the $j$ th evaluation indicator, and $x(i, j)$ is the nonlinear normalized value of indicator $j$ of sample $i$. The standard deviation of all sample projection values is $S(a)=$ $\sqrt{\left\{\sum_{i=1}^{n}[z(i)-\bar{z}]^{2}\right\} /(n-1)}$, in which the larger the $S(a)$ value is, the more dispersed the sample projection points are in general. $n$ and $m$ are the number of samples and the number of evaluation indicators, respectively. $\bar{z}$ is the mean value of $z(i)$. Local density is represented by $D(a)=\sum_{i=1}^{n} \sum_{j=1}^{n}\left(R-r_{i, j}\right) \cdot f\left(R-r_{i, j}\right)$, which indicates the local density of the projection points of all sample. The larger the $D(a)$ value is, the greater the local density is. $r_{i, j}=$ $|z(i)-z(j)|$ represents the absolute distance between the sample $i$ and the sample j. $r_{\max }$ is the maximum value of $r_{i, j}$. $R$ is the radius of the projection window, with a reasonable range of $r_{\max } / 5 \leq R \leq r_{\max } / 3 . f\left(R-r_{i, j}\right)$ is a unit step function. It is 1 when $R>r_{i, j}$; otherwise, it is 0 . $k_{4}=E\left[[z(i)-\bar{z}]^{4}\right] / \sigma^{4}$ is the kurtosis coefficient of the sample projection values. The smaller the value of $k_{4}$ is, the smoother the distribution of the projection values is, which makes the sample projection values more dispersed.

\subsection{The Principle and Characters of Parasitism-Predation} Algorithm (PPA) [37]. Model (1) is a very complicated function with constraints on nonlinear equality and inequality, as well as high power and high dimensionality. It is very difficult to get the real optimal of the model (1). As a rule, metaheuristic algorithms that are nature inspired are prominent because of their considerable and efficient performance when it comes to dealing with projection index function that is complex; this has been observed in the past two decades [23-25, 29-34].

The parasitism-predation algorithm (PPA) is inspired by the multi-interactions among cuckoos, crows, and cats, which mimics the interaction between the predator (cats), the parasite (cuckoos), and the host (crows) in the crow-cuckoo-cat system model to overcome the problems of low convergence and the curse of dimensionality of large data. The proposed hybrid framework combines the relative advantages of cat swarm optimization (CSO), cuckoo search 
TABLE 1: The changing of the sensitivity index, respondence index, and vulnerability index of the tourism economy from 2014 to 2018 in provinces.

\begin{tabular}{|c|c|c|c|c|c|c|c|c|c|c|c|c|c|c|c|}
\hline \multirow{2}{*}{ Province } & \multicolumn{5}{|c|}{$S(i)$} & \multicolumn{5}{|c|}{$R(i)$} & \multicolumn{5}{|c|}{$V(i)$} \\
\hline & 2014 & 2015 & 2016 & 2017 & 2018 & 2014 & 2015 & 2016 & 2017 & 2018 & 2014 & 2015 & 2016 & 2017 & 2018 \\
\hline eijing & & & 000 & & & & & & & & & 68 & 47 & & 32 \\
\hline & & & 822 & & & & & & & & & & & & \\
\hline ebei & 506 & .479 & 0.476 & .544 & & & & 522 & & & & & & & \\
\hline hanxi & 523 & & 0.519 & & & & & & & & & & & & \\
\hline ane & 493 & .476 & 0.468 & .463 & & & & & & & & & & & \\
\hline a & 666 & 0.614 & 0.637 & 0.577 & & & & & & & & & & & \\
\hline & & 468 & 0.558 & 2 & & & & & & & & & & & \\
\hline . & & & 0.519 & & & & & & & & & & & & \\
\hline & 0 & & 0.859 & & & & & & & & & & & & \\
\hline angsu & 803 & 0.799 & 0.814 & 0.805 & 0.806 & 0.820 & 0.863 & 0.857 & & 29 & & & 950 & & .868 \\
\hline & & & 0.805 & & & & & & & & & & & & \\
\hline & & & 0.574 & 6 & & & & & & & & & & & \\
\hline & & 2 & 0.676 & & & & & & & & & & & & \\
\hline & & & 0.497 & 0.4 & & & & & & & & & & & \\
\hline & & & 0.739 & 0.7 & & & & & & & & & & & \\
\hline & 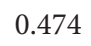 & & 0.554 & & & & & & & & & & & & \\
\hline & & & & & & & & & & & & & & & \\
\hline & & & 0.538 & & & & & & & & & & & & \\
\hline & & & 0.982 & & & & & & & & & & & & \\
\hline & ( & & 0.496 & 0 & & & & 0 & & & & & & & \\
\hline & & & & & & & & & & & & & & & 75 \\
\hline & & & 0.612 & 0.622 & & & & 0.715 & & & & & & & \\
\hline & & & & & & & & & & & & & & & \\
\hline & 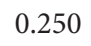 & 0.332 & 0.488 & & & 0. & 0.4 & 0.479 & & & & & 20 & & 0.924 \\
\hline & 25 & 0.458 & 0.435 & & 0.478 & 0. & & 0.460 & 0. & & & & & & 0.885 \\
\hline & 0.572 & 0.439 & 0.449 & 0.465 & 0.471 & 0.266 & 0.250 & 0.288 & 0.313 & 0.313 & 2.149 & 1.755 & 1.561 & 1.488 & 1.503 \\
\hline & & & & & & & & 0.564 & & & & & & & \\
\hline & 78 & .424 & 0.382 & & & & 0 . & 0.381 & . & 65 & & & & & 0.764 \\
\hline & & 0.422 & 0.445 & & & & & 0.423 & & & & & & & 1.077 \\
\hline & 0.432 & 0.475 & 0.460 & 0.454 & 0.551 & 0.535 & 0.514 & 0.497 & 0.491 & & & & 0.926 & 0.923 & 0.987 \\
\hline Xinjiang & 0.521 & 0.530 & 0.557 & 0.585 & 0.577 & 0.416 & 0.397 & 0.396 & 0.442 & 0.473 & 1.253 & 1.338 & 1.405 & 1.324 & 1.219 \\
\hline
\end{tabular}
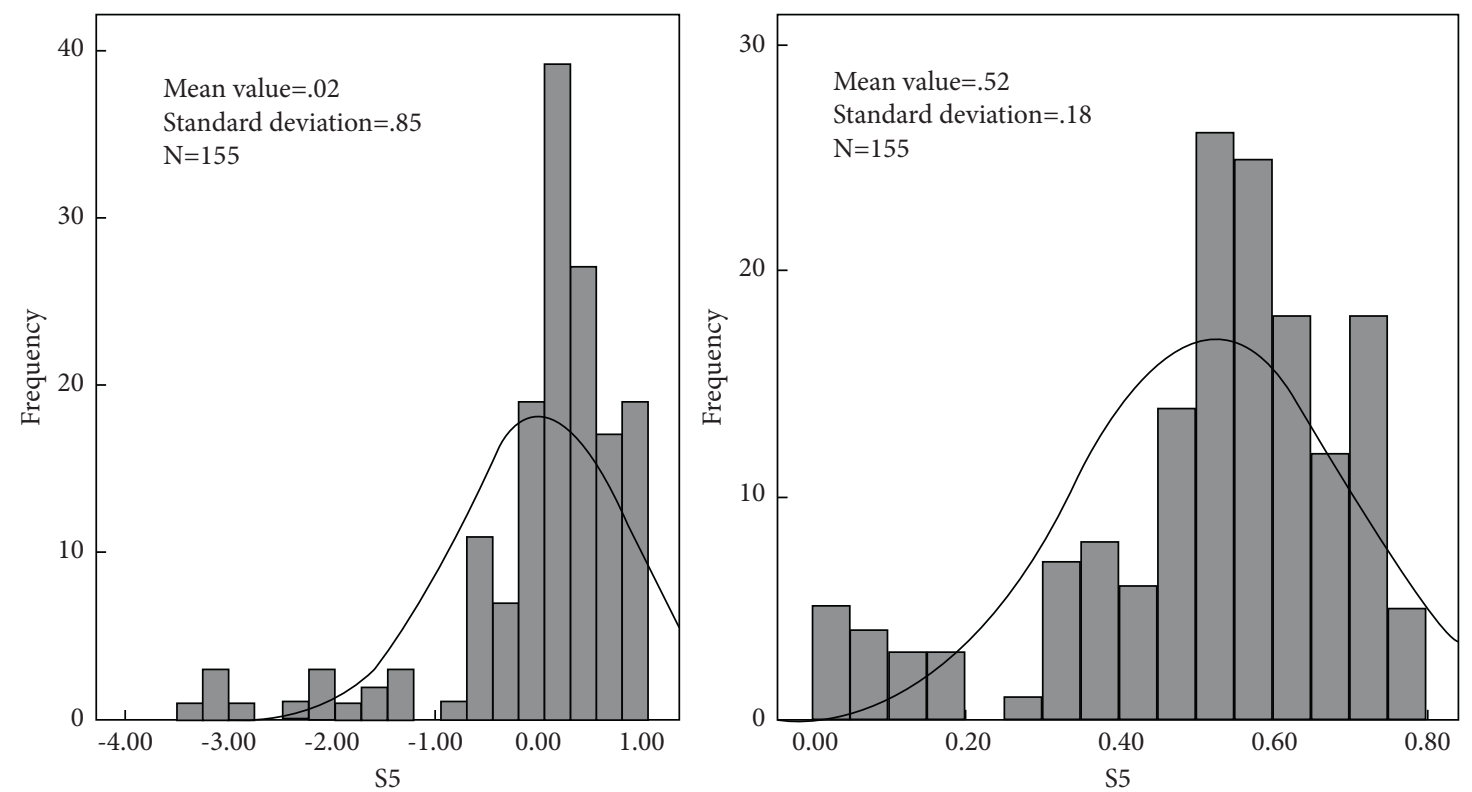

FIgURE 1: Histogram comparison of index S5 before and after sigmoid normalization. 
(CS), and crow search algorithm (CSA) to boost up the accuracy. Nesting, parasitism, and predation phases are supposed to help exploration ability and balance in the context of solving classification problems.

The PPA method is designed and implemented to combine the merits, to overcome the drawbacks of the three optimization algorithms CSO, CSA, and CS, to design an efficient and robust new method which can be faster and better search capability, and to obtain their optimal in various problems. This enables the PPA to make a suitable trade-off between exploration and exploitation abilities.

The experimental results indicate that the PPA is both suitable and useful as a heuristic method and is able to avoid local minima very well compared to CS, CSA, CSA, and WOA algorithms. The PPA is thus used to solve the model (1) in this paper. The more detailed principle and calculation formula are referred to [37].

4.4. The Theorem and the Inference Proposed by Lou et al. $[30,31]$ to Judge Whether to Get the Global Optimal of Model (1) or Not. Although the metaheuristic algorithms such as PPA possess the search capability and get their optimal, there is no guarantee that the global optimal will be reached every time. Lou et al. $[30,31]$ proposed three theorems and two inferences to judge whether the optimization process reaches the real global optimal or not, which are as follows.

Theorem 1. If an indicator is with negative normalization mode and positive mode, its optimized weights must be opposite value with the real global optimal.

Inference 1. If the weight $a(j)$ is optimal, the weight $-a(j)$ must be optimal too.

Inference 2. If the values of the indicator $p$ are constant, its optimal weight $a(p)$ must be zero.

Theorem 2. If the values of two indicators are totally equal, their optimal weights must be equal too.

Theorem 3. If the constraint is that all indicators' weights must be equal or greater than zeroes, a negative indicator's optimal weight must be zero.

With the above-mentioned three theorems and two inferences, by changing the normalization mode of some indicators or adding some dummy indicators with equal values, we can judge whether the optimization process reaches the real global optimal or not. The more detailed proof processes are referred to $[30,31]$.

\subsection{To Establish a PPC Model for Comprehensive Evaluation of} Tourism Economy Vulnerability in Provinces. Two PPC models were established for the sensitivity index and the respondence index, respectively.

Firstly, the nonlinear normalized data of the above 20 sensitivity indicators are imported into the PPC program based on the parasitism-predation algorithm (PPA) [37].
According to the theorems and inferences proposed by Lou et al. $[30,31]$ and taking $R=r_{\max } / 5$, the global optimal solution of the PPC model is obtained, and the optimal weights of the indicators are $\vec{a}\{a(1), a(2), \ldots, a(20)\}=$ $\{-0.2541,-0.0182,-0.0859,0.3031,-0.3370,0.1231,0.2815$, $0.1540,-0.1159,0.3174,0.2843,0.2444,-0.1127,-0.0229$, $-0.2356,-0.4031, \quad-0.0611,0.1937,-0.0034,0.2773)$, $Q(a)=363.51, S(a)=0.3713, D(a)=2680.26, R=0.3659$, $r_{\max }=1.8294$, and $k_{4}=2.7381$. The sensitivity index value (PPC projection value) of each province from 2014 to 2018 is calculated and obtained. From the optimal weights of the indicators, it can be seen that the weights of 12 indicators $(S(1)-S(3), S(5), S(9), S(13)-S(17), S(19)$ are less than zero, indicating that these 12 indicators are "positive indicators" in theory, but in reality, these indicators are negatively correlated with other indicators. This indicates that the "theoretical attribute" of these indicators is inconsistent with their "actual attribute." That is, the smaller the value of the evaluation indicator $S(1)$ is, the greater the sensitivity index is. On the contrary, the value of the evaluate indicator $S(4)$ must be larger. Therefore, for the sensitivity index, the "theoretical attribute" of the evaluation indicators $S(1)$ and $S(4)$ is positive. However, there is a negative correlation between the two indicators' data. According to the correlation analysis results, it can be seen that the indicator $S(16)$ with the largest weight has a significant negative correlation with indexes $S(4), S(6)-S(8), S(10)$, and $S(18)-S(20)$. Also, there is a significant positive correlation between $S(16)$ and $S(3), S(5), S(13)$, and $S(15)$. Therefore, by the established PPC model, we can reliably analyze and judge the "actual attribute" relationship among different indicators and put forward more targeted measures and suggestions to reduce the tourism economy vulnerability in provinces.

Similarly, the nonlinear normalized data of the above 20 respondence evaluation indicators are imported into the PPC program and taking $R=r_{\max } / 5$. We obtain the indicators' optimal weights being $(0.1882,-0.2333,0.0448$, $0.0946,0.1281,0.1922,-0.2560,0.0618,0.3272,0.2350$, $0.1823,-0.1368,0.3289,-0.3146,0.1004,0.3723,0.0556$, $0.2832, \quad 0.2480,0.2481), \quad Q(a)=635.28, \quad S(a)=0.5153$, $D(a)=3245.55, R=0.4739, r_{\max }=2.3694$, and $k_{4}=2.6328$. The respondence index of each province from 2014 to 2018 is calculated and obtained. It can be seen that only the weights of four indicators $(R(2), R(7), R(12), R(14))$ are less than zero, indicating that the "theoretical attribute" of these four indicators is inconsistent with their "actual attribute."

For the inconsistency between "theoretical attribute" and "actual attribute," the optimal weights being less than zero correctly reflects its characteristics.

4.6. To Obtain the Tourism Economy Vulnerability Index in Provinces Based on the Established PPC Model. Some sensitivity indexes and respondence indexes above-obtained are greater than 0 , some are less than 0 , and the others are almost equal to 0 . If the vulnerability index is calculated directly using $V(i)=S(i) / R(i)$, the value of $V(i)$ might be seriously distorted. For example, when $S(i)=0.5$ and $R(i)=0.05$, 
$V(i)$ will amount to 10 , while when $S(i)=0.9$ and $R(i)=0.45, V(i)$ will only be 2 . However, the differences between $S(i)$ and $R(i)$ in both cases are 0.45 . Therefore, in order to maintain generality, the values of $R(i)$ and $S(i)$ should be uniformly and linearly transformed into the range of $[0.25,1.0]$. This gives $V(i) \in[0.25,4]$, which is easier to be classified and understood. The values of sensitivity and respondence index linearly transformed into the range of $[0.25,1]$ are shown in columns " $S(i)$ " and " $R(i)$ " of Table 1 , and the vulnerability index is shown in column " $V(i)$ " of Table 1.

It can be seen from the $S(i)$ values shown in Table 1 from 2014 to 2018 that the sensitivity indexes of $11,12,11,12$, and 13 provinces such as Beijing and Shanghai were higher than the average level, respectively. The respondence indexes of $11,12,11,13$, and 13 provinces such as Beijing and Shanghai were higher than the average level, corresponding to the vulnerability indexes of $15,13,11,14$, and 12 provinces such as Guangdong and Sichuan which are higher than the average level. The vulnerability indexes of $18,14,13,13$, and 10 provinces, respectively, from 2014 to 2018, were greater than 1. These studied results indicate that more and more provinces take the tourism industry as a pillar industry, aim to promote green development, and have gained greater momentum as the idea that "lucid waters and lush mountains are invaluable assets." As a result, most provinces have improved the tourism economy vulnerability, either taking measures with low sensitivity indexes or improving their respondence remarkably, embarking on a relatively healthy road of green growth development of tourism economy. However, a few provinces such as Tibet, Xinjiang, Guangdong, and Hainan have high vulnerability index, which puts great pressure on the balanced development of the tourism industry and the protection of the ecological environment.

\section{Results and Analysis}

\subsection{The Analysis of the Spatiotemporal Differentiation Characteristics of Tourism Economy Vulnerability, Sensitivity, and Respondence of Provinces}

5.1.1. To Embody Differentiation Characteristics with Different Levels. For convenience, the sensitivity, respondence, and vulnerability index are classified into five levels: low, relatively low, medium, relatively high, and high. The sensitivity index $(S)$, respondence index $(R)$, and vulnerability index $(V)$ from 2014 to 2018 were classified by the optimal segmentation method with ranked samples [36] into five levels. The comparison between the classification results of 2014 and 2018 year is shown in Figure 2, and that of 2018 is shown in Table 2.

As for the sensitivity index, most provinces were at low and medium levels in 2014 (in the early stage) and at medium and high levels in 2018 (in the late stage), indicating that most provinces pay attention to improving the tourism economy sensitivity. This is especially true in Hainan, changing from "low" to "relatively high" level. For Hainan province, the values of four important indicators changing greatly thus lead to its sensitivity changing obviously. For example, the two positive indicators are as follows: the proportion of output value of the tertiary industry to GDP (\%) changes from $58.1 \%$ to $69.5 \%$ and the proportion of tourism foreign exchange earnings to total tourism earnings (\%) changes from $51.4 \%$ to 66.3 ; the actual negative indicators are as follows: the proportion of economic losses from natural disasters (\%) changes from $68.8 \%$ to $6.4 \%$ and the proportion of the population affected by natural disasters (\%) changes from $5.07 \%$ to $0.12 \%$. In Guizhou and Ningxia, it promoted from "low" to "medium" level. Most provinces were promoted by one level, except for Liaoning, which changed from "high" to "medium." Overall, the number of $15,37,46,26$, and 27 samples are at "low," "relatively low," "medium," "relatively high," and "high" levels, respectively. This accounts for 9.6\%, 23.9\%, 29.7\%, 17.4\%, and $19.4 \%$, respectively. Hence, the number of samples at "medium" and "relatively low" sensitivity levels is the greatest, the number of samples at a "low" sensitivity level is the smallest, and the number of samples at "relatively high" and "high" sensitivity levels is basically the same.

The distribution characteristic of the respondence index is basically similar to that of the sensitivity index. Most provinces had "low" and "medium" respondence levels in 2014 and "medium" and "high" levels in 2018, indicating that the tourism economy respondence of most provinces has been promoted rapidly, especially that of Hunan, changing from "relatively low" to "relatively high" level, and 11 provinces such as Gansu and Xinjiang have promoted by one level. There are no provinces whose respondence drops by one level or more.

There are 16, 36, 46, 26, and 27 samples with "low," "relatively low," "medium," "relatively high," and "high" respondence levels, accounting for $10.3 \%, 23.2 \%, 29.7 \%$, $17.4 \%$, and $19.4 \%$ respectively, which show that most samples are located at "medium" and "relatively low" respondence levels, the number of samples with "low" respondence is the smallest, and the number of samples with "relatively high" and "high" respondence levels is basically the same.

In terms of tourism economy vulnerability index, in 2014, 15 provinces such as Hebei were at a "relatively high" level, while 6 provinces such as Guizhou and other seven provinces such as Shanghai were at "low" and "relatively low" levels, respectively, and only Tibet was at "high" level. In 2018, only six provinces were at a "relatively high" level, and 10, 9, and 5 provinces were at "low," "relatively low," and "medium" levels, respectively. The results indicate that the tourism economy vulnerability of most provinces has been dropped, especially in Gansu, Anhui, Liaoning, and Hunan provinces, rapidly changing from "relatively high" to "relatively low." Only that of Guizhou has promoted from "low" to "relatively low" level.

There are 30, 49, 39, 29, and 8 samples at "low," "relatively low," "medium," "relatively high," and "high" vulnerability, accounting for $19.3 \%, 31.6 \%, 25.2 \%, 18.7 \%$, and $5.2 \%$, respectively. The results show that most samples are at "medium" and "relatively low" vulnerability levels, the number of samples at "low" and "relatively high" vulnerability is basically the same, and the number of samples at 

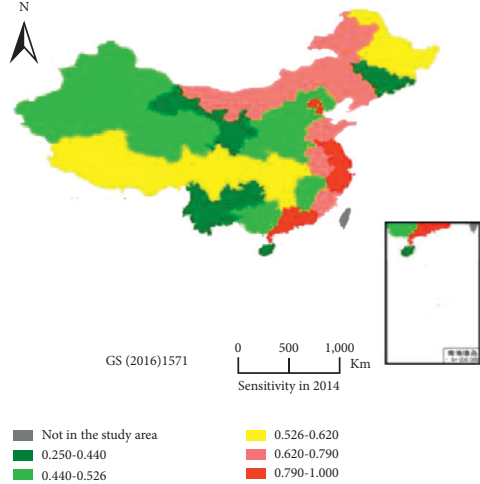

믄. $0.250-0.440$

$0.020-0.790$
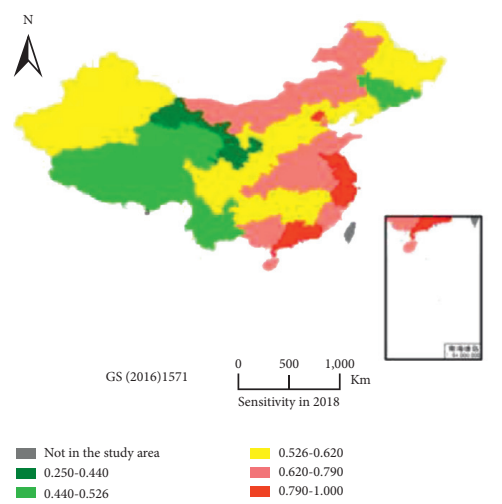
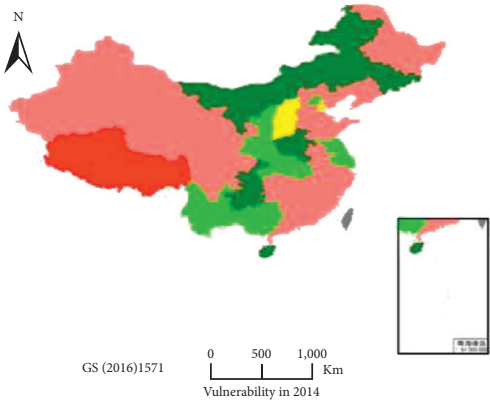

$=0.981-1.065$

다. $1.065-1.330$

(a)

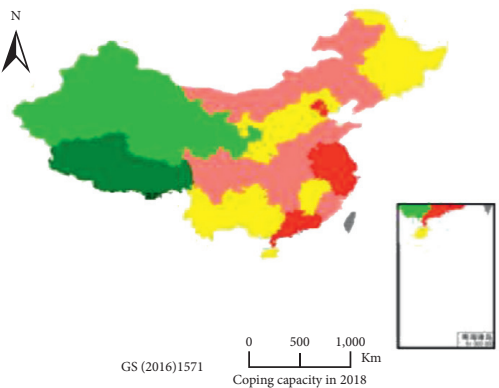

Not in the study are

므 $0.250-0.442$
$0.515-0.605$
$0.605-0.755$
$0.75-1500$

미 $0.605-0.755$

b)

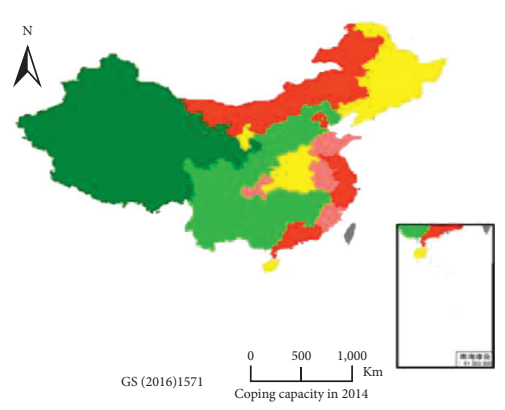

Not in the study area $0.515-0.605$ 므 $0.250-0.442$

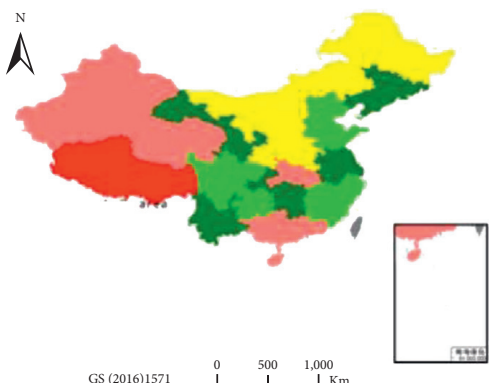

Not in the study area $0.0 .981-1.065$ $\begin{array}{ll}0.540-0.897 & 1.065-1.330 \\ 0.897-0.981 & 1.330-2.200\end{array}$

(b)

FIgURE 2: The comparison of the sensitivity, respondence, and vulnerability index of all provinces in 2014 (a) and 2018 (b).

TABLE 2: Classification results and comparison of sensitivity, respondence, and vulnerability index of provinces in 2018.

\begin{tabular}{|c|c|c|c|c|c|}
\hline & I (low) & II (relatively low) & III (medium) & IV (relatively high) & V (high) \\
\hline S & Gansu & $\begin{array}{l}\text { Tibet, Yunnan, Inner } \\
\text { Mongolia, Qinghai, } \\
\text { and Jilin }\end{array}$ & $\begin{array}{l}\text { Jiangxi, Guizhou, Ningxia, } \\
\text { Hebei, Liaoning, Hunan, Shanxi, } \\
\text { Xinjiang, Heilongjiang, Shaanxi, } \\
\text { and Sichuan }\end{array}$ & $\begin{array}{l}\text { Chongqing, Anhui, } \\
\text { Hainan, Fujian, } \\
\text { Guangxi, Henan, } \\
\text { Shandong, and Hubei }\end{array}$ & $\begin{array}{l}\text { Tianjin, Jiangsu, } \\
\text { Zhejiang, Beijing, } \\
\text { Shanghai, and } \\
\text { Guangdong }\end{array}$ \\
\hline $\mathrm{R}$ & Tibet & $\begin{array}{c}\text { Gansu, Qinghai, and } \\
\text { Xinjiang }\end{array}$ & $\begin{array}{l}\text { Yunnan, Shanxi, Guangxi, } \\
\text { Ningxia, Jilin, Inner Mongolia, } \\
\text { Heilongjiang, Guizhou, Jiangxi, } \\
\text { Shaanxi, Hainan, Hebei, and } \\
\text { Sichuan }\end{array}$ & $\begin{array}{c}\text { Hunan, Hubei, } \\
\text { Liaoning, Henan, } \\
\text { Fujian, Shandong, } \\
\text { Chongqing, and Anhui }\end{array}$ & $\begin{array}{l}\text { Tianjin, Guangdong, } \\
\text { Zhejiang, Beijing, } \\
\text { Jiangsu, and } \\
\text { Shanghai }\end{array}$ \\
\hline $\mathrm{V}$ & $\begin{array}{l}\text { Gansu, Chongqing, Anhui, } \\
\text { Liaoning, Shanghai, } \\
\text { Jiangsu, Inner Mongolia, } \\
\text { Hunan, Yunnan, and Jilin }\end{array}$ & $\begin{array}{l}\text { Jiangxi, Zhejiang, } \\
\text { Tianjin, Guizhou, } \\
\text { Beijing, Hebei, Fujian, } \\
\text { Shandong, and } \\
\text { Sichuan }\end{array}$ & $\begin{array}{l}\text { Ningxia, Shaanxi, Heilongjiang, } \\
\text { Henan, and Shanxi }\end{array}$ & $\begin{array}{c}\text { Hainan, Qinghai, } \\
\text { Guangdong, Hubei, } \\
\text { Xinjiang, and Guangxi }\end{array}$ & Tibet \\
\hline
\end{tabular}

"high" vulnerability is the smallest. Eight samples are at "high" vulnerability, that is, Xinjiang from 2015 to 2017 and Tibet from 2014 to 2018 .

\subsubsection{To Embody Spatiotemporal Distribution Characteristics} with Agglomeration. The sensitivity index has a strong regional agglomeration. For example, in 2014 and 2018, among 10 provinces in East China, except Hebei and Hainan, six provinces such as Beijing and Shanghai were at "high" sensitivity, and Fujian and Shandong were at "relatively high" sensitivity; six provinces in Central China including Shanxi were generally at "relatively low"-"medium" sensitivity in 2014, and at "medium"-"relatively high" sensitivity in 2018; 12 provinces in Western China, except Gansu with low sensitivity, were generally with "low"-"relatively low" sensitivity in 2014 and "relatively low"- "medium" sensitivity in 2018.

Similar to the sensitivity index, among the 10 provinces in Eastern China, eight provinces (except Hebei and Hainan 
being at "relatively low") were at "relatively high" and "high" respondence. Three provinces in Northeast China were at "medium" and "relatively high" respondence. Tibet, Xinjiang, Qinghai, and Gansu were at "low" and "relatively low" respondence. Other provinces in Western and Central China were at "relatively low"-"medium" respondence in 2014 and at "medium"-"relatively high" respondence in 2018.

There are obvious spatiotemporal differences among the three types of indexes. Tibet is at high vulnerability; Xinjiang, Gansu, Qinghai, and some other provinces are at "relatively high" vulnerability. In 2014, five provinces in Eastern China, such as Hebei, Zhejiang, Guangdong, Shandong, and Fujian, are at "relatively high" vulnerability, as well as the provinces in Northeastern China, including Liaoning and Heilongjiang, and provinces in Central China, including Hunan, Jiangxi, and others. In 2018, the provinces in Eastern China such as Hainan and Guangdong were also at "relatively high" vulnerability. Except Guangdong and Hainan provinces, the other eight provinces in Eastern China were at "low" and "relatively low" vulnerability.

\subsection{The Analysis of Indicators' Importance and Ranking}

5.2.1. The Importance and Ranking of Sensitivity Evaluation Indicators. Based on the sensitivity index $z_{S}(i)=\sum_{j=1}^{m} a_{S}(j) S(i, j)$, the greater the optimal weight $a_{S}(j)$ of the indicator $j$, the greater the impact of the indicator $j$ on the sensitivity index is. Therefore, among the 20 sensitivity evaluation indicators, the indicator $S(16)$ has the greatest impact, and its optimal weight is 0.4031 , followed by $S(5)$ and then $S(10)$. After that, we have $S(4)>S(11)>S(7)>S(20)>S(1)>S(12)>S(15)>S(18)>$ $S(8)>S(6)>S(9)>S(13)>S(3)>S(17)>S(14)>S(2)>$ $S(19)$. The weights of the last three indicators are insignificant enough to ignore. The weights of the first four indicators are greater than 0.30 , confirming that they are the most important ones. The weights of the 5 th-10th indicators are between 0.20 and 0.30 , indicating their importance. The weights of the 11th-15th indicators are between 0.10 and 0.20 , indicating their moderate importance. The weights of the 16th-17th indicators are between 0.05 and 0.10 , indicating that they are secondary ones. Finally, the last three indicators are unimportant and can be deleted.

5.2.2. The Importance and Ranking of the Respondence Evaluation Indicators. Among the 20 respondence evaluation indicators, the most important one is the indicator $R$ (16), followed by $R(13)$ and then $R(9)$. After that, we have $R(14)>R(18)>R(7)>R(20)>R(19)>R(10)>R(2)$ $>R(6)>R(1)>R(11)>R(12)>R(5)>R(15)>R(4)>R(8)$ $>R(17)>R(3)$. The top four are the most important indicators, the 5 th to 10 th are important indicators, the 11 th to 16th are moderately important indicators, and the last four are secondary important indicators. The ratio of maximum to minimum weight is less than 9 , indicating that all evaluation indicators are important.
5.2.3. The Importance and Ranking of the Vulnerability Evaluation Indicators. From the calculation formula of vulnerability index $V=S / R$, it can be seen that the more important indicators for sensitivity index and respondence index are also the more important ones for vulnerability index. However, because there is a "quotient" relationship between $S$ and $R$, increasing or decreasing the value of $R$ has a more obvious effect on reducing or increasing the vulnerability index. If the difference of weights between the sensitivity and the respondence indicator is small, the respondence indicator has a greater impact on the vulnerability index. Therefore, provinces should try to take more targeted measures to improve respondence indicator and thus to reduce the vulnerability index more conductively. Specifically, we should first take measures to improve the respondence indicators with large weight such as the ratio of industrial solid waste utilized and urbanization rate. For economically developed provinces such as Guangdong, while vigorously developing the economy, the government must take effective measures to improve their respondence.

\section{Conclusion and Discussions}

\subsection{Conclusion}

6.1.1. Distribution Characteristics of Spatiotemporal Differences among Tourism Economy Vulnerability, Sensitivity, and Respondence. From 2014 to 2018, the tourism economy vulnerability of 31 provinces in China has improved year by year, indicating that all provinces' government has achieved good results in basing themselves on the new development stage, carried out the new development concept, set up a new development pattern, and thus broke a new path to implement high-quality development since the 18th National Congress of the Communist Party of China.

At the same time, the studied results show that there are obvious development imbalance and "agglomeration" characteristics of the tourism economy vulnerability among the provinces in Eastern, Northeast, Central, and Western China. Generally speaking, the overall vulnerability of the provinces in Eastern China is at a low level, and that of provinces in Northeast, Central China, and some provinces in Western China is at a medium level. Although the sensitivity index of provinces in Western China is not at a high level, their respondence is at a relatively low level, resulting in relatively high tourism economy vulnerability. For example, Tibet, Xinjiang, Qinghai, and Gansu provinces are at "high" vulnerability. Therefore, when vigorously developing tourism economy, the provinces' government in Western China must pay more attention to the protection of the ecological environment, consider the construction of roads and other infrastructures, strengthen traffic management, and reduce casualties and direct economic losses caused by accidents. Otherwise, they will be at "high" vulnerability for a long time, which will inevitably lead to the rapid recession of the tourism economy. The vulnerability of the provinces in Central China, Northeast China, and some provinces in Western China is basically at "relatively low" and "medium" vulnerability. These provinces must take precautions to 
realize the high-quality coupling and coordinated development of economy, society, and ecological environment. For provinces in Eastern China, except Guangdong and Hainan, they are basically in the state of "low" and "relatively low" vulnerability, indicating that remarkable results have been achieved in the full implementation of the new development concept. While vigorously developing the tourism economy, provinces such as Guangdong and Hainan must pay more attention to improving their respondence. Otherwise, the high-quality development of the tourism economy will become a tree without a root or water without resource, and the tourism resources will soon be exhausted. In short, provinces' government in both economically developed and developing regions should implement the new development concept and unswervingly take the road of green development with ecological protection as the priority.

In terms of sensitivity and respondence, on the one hand, there is imbalance and "agglomeration," and on the other hand, there is the difference in the distribution of spatiotemporal differentiation characteristics. Overall, the sensitivity and the respondence of provinces in Eastern China are at a relatively high level, those in Western China are at a relatively low level, and those in Northeast China, Central China, and some provinces in Western China are at medium level, which is basically opposite to the distribution characteristics of the vulnerability index. For example, Tibet and Qinghai are at relatively low sensitivity and respondence but at the highest vulnerability.

\subsubsection{The Importance and Ranking of the Evaluation Indicators}

(1) Sensitivity evaluation indicators: Natural disasteraffected population/total population, industrial diversification index, number of traffic accidents, and tourism foreign exchange earnings/tourism earnings are the four most important indicators. Six indicators, including the number of traffic accident casualties, are important indicators. Tertiary industry added value/GDP and some other indicators are medium important indicators. Therefore, in practice, all provinces should first strengthen the prediction, early warning, and prevention of natural disasters, reduce the affected population as much as possible, strengthen and optimize traffic control, and strengthen extreme weather forecast so as to effectively reduce the number of traffic accident deaths and injuries and property losses.

(2) Respondence evaluation indicators: The ratio of industrial solid waste utilized, urbanization rate, the density of grade highway and railway, and the number of healthcare institutions are the four most important indicators, and six indicators, including per capita disposable income of urban households, public service expenditure/fiscal expenditure, and the number of personnel of environmental protection institutions, are important indicators. In practice, provinces should first take measures to improve the ratio of industrial solid waste utilized, promote the development of urbanization, and speed up the construction of roads and other infrastructures.

(3) Vulnerability evaluation indicators: Based on the vulnerability index $V=S / R$, in order to effectively reduce the vulnerability of the tourism economy, all provinces should consider and take comprehensive measures to improve respondence and reduce sensitivity. In particular, priority should be given to improving respondence.

6.1.3. The Establishment of PPC Model for Tourism Economy Evaluation of the Sensitivity, Respondence, and Vulnerability. Based on Ma et al. [19], the evaluation index system of tourism economy sensitivity, respondence, and vulnerability is set up, and the data are normalized with the mean zero and the variation one and sigmoid normalized to improve the distribution of indicator data and eliminate the negative impact of different dimensions on the modeling results, so as to make the modeling results more reliable, stable, and reasonable.

An improved PPC model is established to comprehensively model and evaluate the sensitivity and respondence of the tourism economy in 31 provinces from 2014 to 2018 and calculate the vulnerability index using $V=S / R$. The empirical research results show that, from 2014 to 2018, all provinces have well implemented the new development concept, set up a new development pattern, achieved coordinated, green, and high-quality development of economy, society, and ecology, reduced the vulnerability of the tourism economy to a certain extent, and laid a good foundation for the sustainable development of tourism economy. However, there are still unbalanced problems overall. In terms of sensitivity and respondence, this research shows the characteristics of temporal and spatial differentiation: those of provinces in Eastern China are at a high level, those of provinces in Western China such as Tibet are at a low level, and those of provinces in Central China, Northeast China, and some provinces in Western China are at medium level. The vulnerability distribution characteristics are just the opposite. Most provinces in Western China such as Tibet are at "high" and "relatively high" vulnerability levels, the provinces in Eastern China (except Guangdong and Hainan) are at "low" and "relatively low" vulnerability levels, and some provinces in Northeast China, Central China, and Western China are at "relatively low" and "medium" vulnerability levels.

6.2. Discussions. The research on tourism economy vulnerability can reveal and help judge the main restrictive factors affecting the high-quality development of tourism, which has important theoretical significance and practical value for continuously promoting the high-quality development of tourism under the background of global tourism. Current research methods mainly manage to construct sensitivity evaluation index and respondence evaluation 
index, respectively, and then obtain sensitivity index $(S)$ and respondence index $(R)$, so as to obtain vulnerability index by using $V=S / R$ or $V=a_{0}+a_{1} S+a_{2} R$.

Different scholars have different algorithms for solving the values of sensitivity index $(S)$ and respondence index $(R)$. The main methods used include the set pair analysis method, IEW-SPA-based on information entropy weight, linear weighting method based on information entropy weight, and BPNN. These methods have different principles; thus, the results are bound to be different. As above-mentioned, the information entropy weight cannot reveal the negative correlation between the actual data of the indicator, and the set pair analysis (SPA) has some defects such as asymmetry. The BPNN model has the ability of nonlinear approximation in theory, but the demand of adequate samples must be met, a network structure as compact as possible must be adopted, and the validation samples must be used to monitor the training process to avoid overtraining; otherwise, the established BPNN model might have no generalization ability or practical value.

\section{Data Availability}

The data used were taken from China's annual statistical yearbooks and are available upon request.

\section{Conflicts of Interest}

The authors declare that they have no conflicts of interest.

\section{References}

[1] P. Guillaumont, "Assessing the economic vulnerability of small island developing states and the least developed countries," Journal of Development Studies, vol. 46, no. 5, pp. 828-854, 2010.

[2] D. Navarro-Drazich and C. Lorenzo, "Sensitivity and vulnerability of international tourism by covid crisis: South America in context," Research in Globalization, vol. 3, Article ID 100042, 2021.

[3] F. Gutierrez, E. Barocio, F. Uribe, and P. Zuniga, "Vulnerability analysis of power grids using modified centrality measures," Discrete Dynamics in Nature and Society, vol. 2013, Article ID 135731, 11 pages, 2013.

[4] P. Wei, Y. Peng, and W. Chen, "Climate change vulnerability and key adaptation trajectory of the regional economic system," Discrete Dynamics in Nature and Society, vol. 2021, Article ID 5540452, 14 pages, 2021.

[5] I. Mansouri, J. Hu, K. Shakeri, S. Shahbazi, and B. Nouri, "Assessment of seismic vulnerability of steel and RC moment buildings using HAZUS and statistical methodologies," Discrete Dynamics in Nature and Society, vol. 2017, Article ID 2698932, 16 pages, 2017.

[6] D. Du and P. Ng, "The impact of climate change on tourism economies of Greece, Spain, and Turkey," Environmental Economics and Policy Studies, vol. 20, no. 2, pp. 431-449, 2018.

[7] S. van der Veeken, E. Calgaro, L. Munk Klint et al., “Tourism destinations' vulnerability to climate change: nature-based tourism in Vava'u, the Kingdom of Tonga," Tourism and Hospitality Research, vol. 16, no. 1, pp. 50-71, 2016.
[8] M. Csete, T. Pálvölgyi, and G. Szendrő, “Assessment of climate change vulnerability of tourism in Hungary," Regional Environmental Change, vol. 13, no. 5, pp. 1043-1057, 2013.

[9] F. Li, "Study of vulnerability measurement of Chinese tourism economic system: based on SPA," Tourism Science, vol. 27, no. 1, pp. 15-28, 2013.

[10] Y. Chen and G. Wang, "Analysis on economic system vulnerability of coastal tourism city based on set pair analysis," Geography and Geo-Information Science, vol. 29, no. 5, pp. 94-97, 2013.

[11] F. Li, N. Wan, B. Shi, X. Liu, and Z. Guo, "The vulnerability measure of tourism industry based on the perspective of "environment-structure" integration A case study of 31 provinces in mainland China," Geographical Research, vol. 33, no. 3, pp. 569-581, 2014.

[12] B. Lu, Q. Ming, X. Guo, and T. Li, "Vulnerability evaluation, influencing factors and coping strategies of tourism economic system in frontier provinces - a case study of Yunnan," Journal of Yunnan Normal University (Natural Sciences Edition), vol. 38, no. 5, pp. 66-74, 2018.

[13] Y. Yang, B. Shi, and X. Liu, "Research on the regional economic vulnerability evaluation under background of tourism economy dependence- taking Zhangjiajie, Aba and Lijiang as examples," The Theory and Practice of Finance and Economics, vol. 40, no. 5, pp. 128-135, 2019.

[14] F. Su, Y. Chen, and P. Zhang, "Vulnerability assessment of tourism city's economic system based on the set pair analysis: a case study of Zhoushan city," Scientia Geographica Sinica, vol. 33, no. 5, pp. 538-544, 2013.

[15] Y. Yang and R. Wang, "Research on vulnerability assessment of tourism economic system and the path optimization in border ethnic region: a case study of Korean autonomous prefecture of Yanbian," Areal Research and Development, vol. 34, no. 6, pp. 104-109, 2015.

[16] G. Weng and Q. Zhang, "Vulnerability evaluation of the tourism economic system in western regions," Commercial Research, vol. 7, pp. 185-192, 2015.

[17] F. Jia, H. Miao, Y. Kong, and Y. Geng, "Evaluation on system vulnerability of regional tourism economy in Ningxia," Journal of Ningxia University (Natural Science Edition), vol. 38, no. 4, pp. 409-414, 2017.

[18] Q. He, Y. Li, and K. Xu, "Study on tourism economic system's vulnerability assessment of Taiwan area," Territory \& Natural Resources Study, vol. 1, pp. 77-81, 2017.

[19] H. Ma, Q. Lian, Y. Lun, and J. Xi, "Spatial differentiation of tourism economic system vulnerability based on BP neural network in different provinces of China," Resources Science, vol. 41, no. 12, pp. 2248-2261, 2019.

[20] L. Tian, Y. Tian, and Y. Yang, "Study on tourism economic system's vulnerability assessment of Dali prefecture based on TOPSIS," Resource Development \& Market, vol. 33, no. 12, pp. 1529-1534, 2017.

[21] Y Lu, X Li, S Zibibula, and X. Zhao, “Tourism economic system's vulnerability assessment of Hotan prefecture in Xinjiang," Areal Research and Development, vol. 36, no. 1, pp. 120-124, 2017.

[22] X. Xie, "Vulnerability assessment of tourism economic system in the core area of the silk road economic belt," Journal of Xinjiang University (Philosophy, Humanities \& Social Sciences), vol. 47, no. 5, pp. 9-16, 2019.

[23] J. Wang, H. Wu, and T. Yang, "Vulnerability assessment of rainfall and waterlogging in subway stations based on projection pursuit model," China Safety Science Journal, vol. 29, no. 9, pp. 1-7, 2019. 
[24] D. Dong and S. Feng, "Evaluation of community vulnerability to fire hazards based on projection pursuit model," China Safety Science Journal, vol. 28, no. 9, pp. 33-38, 2018.

[25] L. Zhang and X. Chen, "Assessing model of vulnerability for flood control system based on projection pursuit," Water Resources and Power, vol. 28, no. 7, pp. 37-40, 2010.

[26] StatSoft Inc, Electronic Statistics Textbook [EB/OL], StatSoft Inc., Tulsa, OK, USA, 2011, http://www.statsoft.com/ textbook.

[27] W. Lou and L. Qiao, "Early warning model of financial risks and empirical study based on neural network," Finance Forum, vol. 11, pp. 52-61, 2011.

[28] Z. Wang, "Comparative study on the tourism economy vulnerability in developed and developing countries," Academic Review, vol. 1, pp. 30-32, 1989.

[29] W. Lou, R. Gan, and T. Li, "The library outcomes (performances) evaluation using the projection pursuit clustering model," Library and Information Service, vol. 61, no. 9, pp. 65-73, 2017.

[30] W. Lou and L. Qiao, "New theory exploration of projection pursuit clustering model and its positive research," Journal of Applied Statistics and Management, vol. 34, no. 1, pp. 47-58, 2015.

[31] W. Lou, P. Xiong, G. Feng, and X. Yu, "Key factors influencing the projection pursuit clustering technique and its positive research," Journal of Applied Statistics and Management, vol. 36, no. 5, pp. 783-801, 2017.

[32] W. Lou and P. Xiong, "Water quality comprehensive evaluative and prediction of the Yangtze River applying projection pursuit clustering technique and its positive analysis," Journal of Earth Environment, vol. 5, no. 5, pp. 344-352, 2014.

[33] X. Zhang and M. Huang, "Study on vulnerability evaluation method of lightning disaster based on projection pursuit dynamic clustering model," in Proceedings of the 2018 7th International Conference on Energy and Environmental Protection (ICEEP 2018), vol. 170, July 2018.

[34] X. Yu, W. Lou, and H. Kang, "Dynamic clustering projection pursuit modeling for credit risk assessment of the corporates on supply chain and its positive research," Mathematics in Practice and Theory, vol. 48, no. 11, pp. 32-40, 2018.

[35] J. H. Friedman and J. W. Tukey, "A projection pursuit algorithm for exploratory data analysis," IEEE Transactions on Computers, vol. c-23, no. 9, pp. 881-890, 1974.

[36] Q.-Y. Tang and C.-X. Zhang, "Data Processing System (DPS) software with experimental design, statistical analysis and data mining developed for use in entomological research," Insect Science, vol. 20, no. 2, pp. 254-260, 2013.

[37] A.-A. A. Mohamed, S. A. Hassan, A. M. Hemeida, S. Alkhalaf, M. M. M. Mahmoud, and A. M. Baha Eldin, "Parasitism predation algorithm (PPA): a novel approach for feature selection," Ain Shams Engineering Journal, vol. 11, no. 2, pp. 293-308, 2020. 